\title{
Moving fast towards the future: the role of \#SoMe in learning cardiology
}

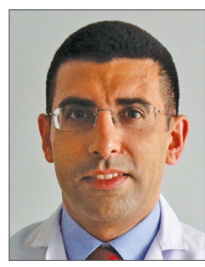

\author{
Salvatore Brugaletta*, $\mathrm{MD}, \mathrm{PhD}$ \\ Cardiovascular Institute, Hospital Clinic, Institut d'Investigacions Biomèdiques August Pi i Sunyer (IDIBAPS), \\ Barcelona, Spain
}

Some years ago, I had to moderate a session at EuroPCR on how social media can be applied in clinical practice. I knew about social media, but not about their potential applicability in my practice. For this reason, I started collecting as much information as possible and I must say that, after an initial scepticism, I was really fascinated by the data collected and by the potential of social media in the learning process.

The usefulness of digital media was highlighted some months ago in a very nice editorial by Davide Capodanno, discussing the educational resources shared online in the form of streaming, webcasts, webinars, slides, etc ${ }^{1}$. Some medical congresses have a number of sessions recorded on site for future website transmissions, and some medical societies, such as the Spanish Society of Cardiology, even have totally virtual congresses with live streaming. Social media are an important part of this digital revolution and represent a dramatic change in traditional healthcare conversations. In the new age of instantaneous global communication, dominated by mobile phone communication, it makes little sense to ignore the insights and perspectives from the easily accessible, worldwide community of practice. Social media platforms, such as
Twitter and Facebook, have emerged as a forum to discuss interesting and challenging cases, medical data, clinical trials, articles published and to share opinions amongst users. Physicians have the opportunity to increase and advance their clinical and scholarly activities by using social media. Social media indeed give the opportunity to do much more than passively digest information, harnessing our new connectivity to foster discussions between clinicians around the world ${ }^{2}$. Twitter has been defined as a flow; conversely, an e-mail inbox is a queue because we have to deal with each message one way or another, even if we simply delete them. On Twitter there is no way to check everything, so we just have to learn to sample the flow, and doing so involves knowing how to focus attention ${ }^{3}$.

Modern interconnectivity can move us from simply reading about novel therapies, treatments, and devices to discussing them directly with the scientists who developed them and with many experts across the world. This engagement between scientists and learned audience members is keeping the scientific community vibrant and active and contributes to the learning of everyone. 
Whereas each hospital has the so-called "Heart Team" to discuss difficult cases, in social media there is a social "Heart Team", with a somehow larger knowledge and experience as it may be composed of high expertise doctors in many specific fields.

Social media is also a forum for medical congresses, allowing attendees to share what they are learning in real time with those who cannot attend physically, and allowing debate of interesting topics and articles. This was the case, for example, of the ORBITA trial ${ }^{4}$, which was extensively debated and criticised on social media, prompting one of the investigators to open a Twitter account to explain the nuances of the study. While criticism of a published article rarely happens on a journal's website, critical commentary of published work happens freely and frequently on Twitter, where participants, not otherwise allowed, may provide perspectives on health-related issues traditionally unrepresented in a medical forum.

Twitter can also be an important source for reading relevant articles, saving time and crowd-sourcing literature reviews for those physicians trying to keep updated with medical literature. The process of sharing and re-tweeting can indeed be used as a natural filter for selecting articles of particular relevance: a user may prioritise, for example, reading articles that have been posted by multiple people, particularly recognised leaders or other users who share their scholarly interests.

Last, but not least, in the year of the 40th anniversary of PCI, social media have recently been used to teach new technical skills. The novel distal left radial approach (\#ldtra) for coronary interventions, recently published in EuroIntervention ${ }^{5}$, was widely discussed and disseminated on social media by Dr Kiemeneij. Many social media users have learnt how to do it only by enjoying the social media discussion and interacting directly with one of the pioneers of this technique.

Ultimately, it is important to be aware of social media risks. The risk of breach of confidentiality must be considered and avoided at all costs. The rules and ethical considerations for publishing clinical images in medical journals should also apply to online social media platforms. The same also applies for sharing data from medical congresses: embargo rules for late-breaking clinical trials and first report investigation should be respected. The interaction between users should always be guided by professionalism and education, avoiding posting when you are angry (online lives forever!). Many national and international healthcare organisations and authorities have developed guidelines either to respect patient information privacy online or to keep physicians within their ethic and deontological code.

For all these reasons, it is easy to understand that social media represent the present and the future of our medical education. In this, the choice of EuroIntervention to have an \#SoMe team is very valuable. I have the pleasure of leading this team (@sbrugaletta), whose aim will be to enhance social media interaction around the journal and to keep the cardiology community updated. We have appointed an international social board team worldwide, composed of Dr Kunadian (@Vijaykunadian; UK), Dr Ortega-Paz (@Ortega_Paz; Spain),Dr Alraies (@chadialraies; USA), Dr Suh (@willsuh76; USA), Dr Alasnag (@mirvatalasnag; Saudi Arabia) and Dr Waqar (@jedicath; Saudi Arabia), in order to create a large social interaction from any geographical regions and to pay attention to the unmet needs of our worldwide community.

There are then no reasons for not opening a social media account and for not joining our social community on Twitter (@EuroInterventio) and Facebook (www.facebook.com/EuroIntervention)!

\section{Conflict of interest statement}

S. Brugaletta reports receiving a research grant to his institution from AstraZeneca, and speaker's fees from Abbott Vascular and Boston Scientific.

\section{References}

1. Capodanno D. Digital speakers in the digital era. EuroIntervention. 2017; 13:379-80.

2. Chan TM, Thoma B, Radecki R, Topf J, Woo HH, Kao LS, Cochran A, Hiremath S, Lin M. Ten steps for setting up an online journal club. J Contin Educ Health Prof. 2015;35:148-54.

3. Rheingold H. Attention, and Other 21st-Century Social Media Literacies. EDUCAUSE Review. 2010;45:14-24.

4. Al-Lamee R, Thompson D, Dehbi HM, Sen S, Tang K, Davies J, Keeble T, Mielewczik M, Kaprielian R, Malik IS, Nijjer SS, Petraco R, Cook C, Ahmad Y, Howard J, Baker C, Sharp A, Gerber R, Talwar S, Assomull R, Mayet J, Wensel R, Collier D, Shun-Shin M, Thom SA, Davies JE, Francis DP; ORBITA investigators. Percutaneous coronary intervention in stable angina (ORBITA): a double-blind, randomised controlled trial. Lancet. 2018;391:31-40.

5. Kiemeneij F. Left distal transradial access in the anatomical snuffbox for coronary angiography (ldTRA) and interventions (ldTRI). EuroIntervention. 2017;13:851-7. 